\title{
Frugivoria e potencial dispersão de sementes pelo marsupial Gracilinanus agilis (Didelphidae: Didelphimorphia) em áreas de Cerrado no Brasil central
}

\author{
Nícholas Ferreira de Camargo ${ }^{1}$, Ramatis Machado Scarponi Cruz², \\ Juliana Fernandes Ribeiro ${ }^{1}$ e Emerson Monteiro Vieira ${ }^{3,4}$
}

Recebido em 16/02/2011. Aceito em 22/06/2011

\begin{abstract}
RESUMO
(Frugivoria e potencial dispersão de sementes pelo marsupial Gracilinanus agilis (Didelphidae: Didelphimorphia) em áreas de Cerrado no Brasil central). No presente estudo, investigamos o consumo de frutos e viabilidade de sementes consumidas pelo marsupial Gracilinanus agilis (Burmeister, 1854) em quatro áreas de cerradão do Brasil central. Análises fecais indicaram que este mamífero se alimenta de plantas pioneiras da família Melastomataceae (Miconia albicans (Sw.) Triana, M. cuspidata Naudin, M. ferruginata DC., M. pepericarpa DC., M. pohliana Cogn. e Ossaea congestiflora (Naudin) Cogn.), Solanaceae (uma espécie não identificada) e Viscaceae (Phoradendron perrottetii (DC.) Eichler, ervade-passarinho). Detectamos o maior índice de consumo de frutos já registrado para o gênero Gracilinanus, com cerca de $45 \%$ das fezes contendo sementes e $86 \%$ contendo itens derivados de frutos ( $\mathrm{N}=422)$. Com exceção de O. congestiflora, o restante das sementes das espécies testadas não sofreram efeitos negativos na germinabilidade ao passarem pelo trato digestório deste marsupial. A avaliação da variação intraspecífica no número de sementes encontradas nas fezes indicou que fêmeas de $G$. agilis defecam maior quantidade de sementes de Miconia (média $\pm \mathrm{EP}=21,7 \pm 3,8$ ) em comparação com os machos $(14,4 \pm 3,0)(F=26,32 ; P<0,0001)$. O fator estação do ano também foi significativo $(F=452,22 ; P<0,0001)$ e existiu interação entre sexo e estação do ano $(F=30,10 ; P<0,0001)$. Tanto fêmeas como machos aumentaram o consumo de frutos com sementes na época seca, contudo fêmeas apresentaram mais sementes nas fezes do que machos na estação chuvosa. Nessa estação, observamos um maior número de sementes de espécies de Miconia defecadas por fêmeas reprodutivas $(24,7 \pm 6,6)$ em relação a fêmeas não reprodutivas $(12,9 \pm 2,8)(P=0,031)$. Nossos dados indicaram que G. agilis selecionou positivamente espécies da família Melastomataceae, sugerindo um papel relevante dessas plantas na dieta desse marsupial, relacionado ao suprimento da demanda energética e de água, principalmente na época seca.
\end{abstract}

Palavras-chave: Cerrado, frutos, germinação, marsupial, Melastomataceae

\begin{abstract}
(Frugivory and potential seed dispersal by the marsupial Gracilinanus agilis (Didelphidae: Didelphimorphia) in areas of Cerrado in central Brazil). In the present study, we investigated patterns of fruit consumption and viability of seeds consumed by the marsupial Gracilinanus agilis (Burmeister, 1854) in four cerrado sites in central Brazil. Faecal analysis indicated that this mammal feeds on seeds of pioneer plant species of Melastomataceae (Miconia albicans (Sw.) Triana, M. cuspidata Naudin, $M$. ferruginata DC., M. pepericarpa DC., M. pohliana Cogn. and Ossaea congestiflora (Naudin) Cogn.), Solanaceae (one unidentified species), and Viscaceae (Phoradendron perrottetii (DC.) Eichler, mistletoe). We recorded the highest reported rate of fruit consumption for Gracilinanus, with about $45 \%$ of the scats containing seeds and $86 \%$ containing items derived from fruits ( N $=422$ ). With the exception of $O$. congestiflora, the remaining seed species tested did not suffer negative effects on germination rates after passing through the marsupial guts. The analysis of intraspecific variation in the total number of Miconia seeds found in scats indicated that females defecate a significantly greater number of seeds (mean $\pm \mathrm{EP}=21.7 \pm 3.8)$ than males $(14.4 \pm 3.0)$ $(F=26.32 ; P<0.0001)$. The factor season was also significant $(F=452.22 ; P<0.0001)$ as well as the interaction between sex and season $(F=30.10 ; P<0.0001)$. Both females and males increased their fruit consumption in the dry season, but in the rainy season females had more seeds than males in their faeces. Later during this season, we observed a higher number of Miconia seeds defecated by reproductive females $(24.7 \pm 6.6)$ compared to non-reproductive females $(12.9 \pm 2.8)(P=0.031)$. Our results indicate that G. agilis feeds selectively on Melastomataceae, which suggests these plants play an important role in the diet of this marsupial in relation to energy and water demands, especially during the dry-cool season.
\end{abstract}

Key words: Cerrado, fruits, germination, marsupial, Melastomataceae

\footnotetext{
1 Universidade de Brasília, Pós-Graduação em Ecologia, Brasília, DF, Brasil

2 Universidade de Brasília, Curso de Ciências Biológicas, Brasília, DF, Brasil

3 Universidade de Brasília, Departamento de Ecologia, Laboratório de Ecologia de Vertebrados, Brasília, DF, Brasil

4 Autor para correspondência: emvieira@unb.br
} 


\section{Introdução}

As interações mutualísticas entre plantas e animais que se alimentam de frutos propiciam a dispersão das primeiras (van der Pijl 1982). Geralmente os frutos possuem como características serem nutritivos e carnosos, atraindo de forma mais eficiente o seu dispersor (van der Pijl 1982). O valor adaptativo desta relação mutualística pode ser inferido pela prevalência na natureza de animais e plantas envolvidos (Jordano 2000). Willson (1993) estimou que 50 a 75\% das árvores de florestas tropicais são dispersas por animais. Como estratégia, algumas plantas também tendem a investir em maior quantidade de sementes por fruto, aumentando assim a probabilidade absoluta de sucesso das mesmas (Wiesbauer et al. 2008). Espécies que apresentam essas características geralmente são plantas de estádios iniciais de sucessão (pioneiras) e apresentam síndrome de dispersão zoocórica mista, realizada tanto por aves quanto mamíferos (Wiesbauer et al. 2008).

O processo de dispersão zoocórica possibilita o aumento da aptidão da planta ao propiciar o deslocamento do propágulo para longe da planta mãe, o que reduz a competição por recursos locais e a taxa de predação potencial por insetos ou roedores (Janzen 1970; Connell 1971; Howe et al. 1985). Além disso, a dispersão aumenta a probabilidade do encontro de novos ambientes mais propícios para a germinação das sementes e estabelecimento da plântula. As sementes consumidas pelos animais dispersores muitas vezes estão intactas no estômago e fezes, viabilizando a germinação a posteriori (Cantor et al. 2010). A germinabilidade das sementes pode inclusive aumentar ao entrar em contato com o trato gastrointestinal do animal, propiciando a escarificação térmica, física ou química, quebrando a dormência das sementes (Clergeau 1992).

Recentemente, alguns integrantes do grupo dos pequenos mamíferos vêm sendo reportados como bons dispersores de sementes (ver Willson 1993; Medellín, 1994; Vander Wall 2002; Pimentel \& Tabarelli 2004; Casella \& Cáceres 2006; Lessa \& Costa 2009). Apesar de alguns roedores atuarem como dispersores (Smythe 1978; Dubost 1988; Forget 1993; Theimer 2001), geralmente agem mais como predadores (Price \& Jenkins 1986). Para os marsupiais, no entanto, como não roem o alimento, há a indicação de um papel mais relevante na dispersão em comparação com os roedores (ver Ballardie \& Whelan 1986; Cowan 1990; Amico \& Aizen 2000; Dungan et al. 2002; Dennis 2003).

Na região neotropical, a dispersão por marsupiais didelfídeos tem sido relatada em alguns estudos (Amico \& Aizen 2000; Casella \& Cáceres 2006; Lessa \& Costa 2009; Cantor et al. 2010), mesmo que o grau de frugivoria varie entre espécies. Apesar de existirem registros de espécies de marsupiais se alimentando de frutos, poucos são os estudos que avaliam estes animais como potencial dispersores de sementes (ver porém Grelle \& Garcia 1999; Cáceres \& Monteiro-Filho 2000; Cáceres 2002; Lessa \& Costa 2009). Contudo, alguns estudos revelaram que as sementes consumidas por algumas espécies apresentam maior grau de germinação após a passagem pelo trato digestório (e.g. Cáceres 2002; Lessa \& Costa 2009; Cantor et al. 2010). Esses trabalhos apontam para a possível relevância destes animais como engenheiros ambientais (sensu Lawton 1994), uma vez que podem influenciar diretamente na dinâmica populacional das plantas locais por meio da dispersão (Lessa \& Costa 2009).

Os marsupiais podem ser importantes dispersores potenciais, especialmente de espécies de plantas pioneiras (Medellín 1994, Lessa \& Costa 2009), apesar de suas especificidades em termos de preferências alimentares. Um dos grupos de marsupiais neotropicais que se alimentam de frutos é composto pelos representantes do gênero Gracilinanus. No entanto, poucos são os estudos publicados que tenham avaliado a dieta de espécies desse gênero. $\mathrm{O}$ estudo mais detalhado até o momento foi conduzido por Martins et al. (2006), investigando a espécie G. microtarus (Wagner, 1854). Apesar dos autores terem registrado o animal consumindo representantes de três gêneros de planta (Miconia, Passiflora e Solanum), não foi avaliado o potencial deste animal como dispersor. Somente um estudo investigou a potencialidade de um representante do gênero - Gracilinanus agilis (Burmeister, 1854) - como dispersor de sementes após o consumo de frutos em cerradão, no estado de Minas Gerais (Lessa e Costa 2009). Além disso, esse foi o único estudo sobre possível dispersão de sementes por marsupiais conduzido no bioma Cerrado. Esse bioma é rico em espécies de mamíferos (Marinho-Filho et al. 2002) e também considerado um dos ecossistemas mais ameaçados do mundo (Myers et al. 2000).

O estudo de Lessa \& Costa (2009), embora importante, não abrangeu tópicos relevantes na diferença do consumo dos itens alimentares em relação a possíveis variações intrapopulacionais, associadas a fatores tais como sexo, época reprodutiva e sazonalidade. Estudos desta natureza são de grande importância para o entendimento da dieta da fauna de forma geral, uma vez tais fatores podem explicar como a espécie pode apresentar diferentes papéis ecológicos. Por exemplo, G. microtarsus apresenta diferenças entre machos e fêmeas em relação ao consumo de insetos (Martins et al. 2006). Entretanto, ainda não foi realizado um estudo que avalie tal variação intraespecífica no consumo de frutos para qualquer espécie de Gracilinanus.

Um possível motivo para a escassez de estudos referentes ao papel de pequenos marsupiais como dispersores pode ser a constatação do pouco consumo de frutos por estes animais (Martins et al. 2006; Lessa \& Costa 2009). No entanto, dois possíveis fatores podem fazer com que o consumo de frutos por esses animais seja subestimado: a alta taxa de digestão da polpa (Caron et al. 1985; Astúa de Moraes et al. 2003) e o ato de cuspir as sementes (Leiner \& Silva 2007). Uma possível avaliação incorreta da importância de frutos na dieta de Gracilinanus foi corroborada por dados obtidos em laboratório, que indicaram um consumo relativamente alto 
de frutos por G. agilis (Astúa de Moraes et al. 2003, Santori \& Astúa de Moraes 2006). Com base nesses dados, Santori \& Astúa de Moraes (2006) caracterizam esse marsupial como frugívoro-onívoro. Uma forma de se avaliar, de forma indireta, o consumo de frutos utilizando dados de campo é a contagem de sementes encontradas em amostras fecais.

O possível papel de G. agilis como dispersor de espécies de Cerrado no Brasil central foi aqui estudado. Os nossos objetivos foram: 1) verificar a viabilidade das sementes encontradas nas fezes de G. agilis; 2) avaliar potenciais variações intraespecíficas (i.e., sexo, condição reprodutiva) e sazonais (i.e., estação seca e chuvosa) no consumo de frutos; 3) investigar uma possível seleção por determinadas espécies (ou grupos) de frutos. Em relação a esses objetivos, as nossas expectativas foram as seguintes: 1) As sementes das espécies de plantas consumidas por G. agilis apresentam maior germinabilidade ao se comparar com sementes retiradas diretamente dos frutos (como verificado por Lessa \& Costa 2009); 2) O consumo de frutos é maior na estação chuvosa, uma vez que a abundância de frutos aparentemente também é maior nessa época do ano (q.v., Batalha \& Martins 2004); 3) Fêmeas se alimentam mais de frutos em comparação aos machos, como reflexo da maior demanda energética quando estas estão reprodutivas; 4) Fêmeas reprodutivas se alimentam mais de frutos em relação às não reprodutivas, uma vez que demanda energética destas é maior, e frutos possuem quantidades relevantes de açúcar (fonte direta de energia para o organismo); 5) Esse marsupial se alimenta dos frutos na mesma proporção em que eles ocorrem na natureza, uma vez que trata-se de um frugívoro aparentemente generalista (Martins et al. 2006; Lessa \& Costa 2009).

\section{Material e métodos}

\section{Espécie estudada}

G. agilis possui uma distribuição geográfica que se estende desde a fronteira do Panamá com a Colômbia até o nordeste, centro-oeste e sudeste do Brasil, com a necessidade de confirmação para esta espécie nos estados do Paraná e Rio Grande do Sul (Rossi et al. 2006). Este marsupial de pequeno porte apresenta massa corporal entre 20 e $30 \mathrm{~g}$ e comprimento de cabeça-corpo entre 90 e 106 mm (Emmons \& Feer 1990). G. agilis apresenta hábitos noturnos (Emmons \& Feer 1990) e possui cauda preênsil (Rossi et al. 2006), adaptação típica de animais que utilizam estratos arbóreos (Rose 1987). Esse didelfídeo está associado geralmente a formações florestais típicas do Cerrado, tais como mata de galeria e cerradão, podendo ser capturado ocasionalmente em cerrado senso estrito e campo úmido (Emmons \& Feer 1990; Rossi et al. 2006). A reprodução é extremamente marcada (Mares \& Ernest 1995), acontecendo nas épocas de maior pluviosidade (Mares \& Ernest 1995; Rossi et al. 2006).

\section{Área de estudo}

Realizamos o estudo em áreas de Cerrado do Brasil central. Esse bioma é considerado o segundo maior bioma Neotropical $\left(2 \times 10^{6} \mathrm{~km}^{2}\right)$, incluindo fitofisionomias savânicas, campestres e florestais reguladas por meio de características climáticas e edáficas do platô do Brasil Central (Eiten 1972). Coletamos os dados na Área de Proteção Ambiental do Gama-Cabeça de Veado (APA-GCV), que inclui cerca de 10.000 ha de áreas protegidas contíguas no Distrito Federal: a Reserva Ecológica do Roncador (RECOR - IBGE), a Estação Ecológica do Jardim Botânico de Brasília (EEJBB) e a Fazenda Água Limpa (FAL), area de conservação e pesquisa da Universidade de Brasília. Essa região é composta por fitofisionomias naturais típicas de Cerrado (Câmara 2008; Fonseca \& Silva Júnior 2004).

Dentro da APA-GCV, selecionamos quatro sítios de amostragem. Todos esses apresentavam a mesma fitofisionomia (cerradão) e nenhum deles distava menos de 3 $\mathrm{km}$ de qualquer outro sítio amostrado. Três sítios estavam localizados no JBB e um na FAL (Fig. 1). O Cerradão é uma formação florestal cuja altura do estrato arbóreo varia entre 8 e $15 \mathrm{~m}$, com uma cobertura vegetal que oscila de $50 \%$ a $90 \%$. O cerradão, apesar de fisionomicamente parecer uma floresta, possui espécies tanto de Cerrado sentido restrito quanto de matas, assemelhando-se floristicamente a um Cerrado (Ribeiro \& Walter 1998). A variação sazonal do Cerrado é representada por duas épocas do ano, seca e chuvosa. A época chuvosa caracteriza-se por ser úmida e quente e ocorre de outubro a abril, já os meses entre abril e setembro são secos e frios, evidenciando a época seca (Eiten 1972).

\section{Métodos de captura}

Capturamos os animais de setembro de 2009 a agosto de 2010 utilizando-se armadilhas do tipo Sherman de dois tamanhos (12,0 cm x 10,0 cm x 37,5 cm e 9,0 cm x 8,0 cm x $23,5 \mathrm{~cm}$ ) em grades quadradas de 144 estações ( 12 x 12) com intervalos de $15 \mathrm{~m}$ entre elas. No interior da grade foram dispostas, de forma aleatória, 80 armadilhas no solo e 80 no sub-bosque, totalizando 160 armadilhas por grade. Estabelecemos quatro grades uma em cada sítio estudado. Em cada uma delas houve três sessões de captura em cada estação do ano (seca e chuvosa). Cada sessão tinha a duração de seis noites, resultando em um esforço de 5.760 armadilhas-noite por sítio e 23.040 armadilhas-noite no total. A isca utilizada foi composta de uma mistura de creme de amendoim, farinha de milho, banana, emulsão scott e essência de baunilha.

Os indivíduos coletados foram anilhados e tiveram registrados o sexo, estado reprodutivo e idade. A determinação dos sexos foi feita a partir da observação direta da genitália. Verificamos as condições reprodutivas principalmente pelo inchaço das mamas nas fêmeas (Pinheiro et al. 2002). No caso dos machos, pela impossibilidade de se determinar com confiança a condição reprodutiva com base nas 


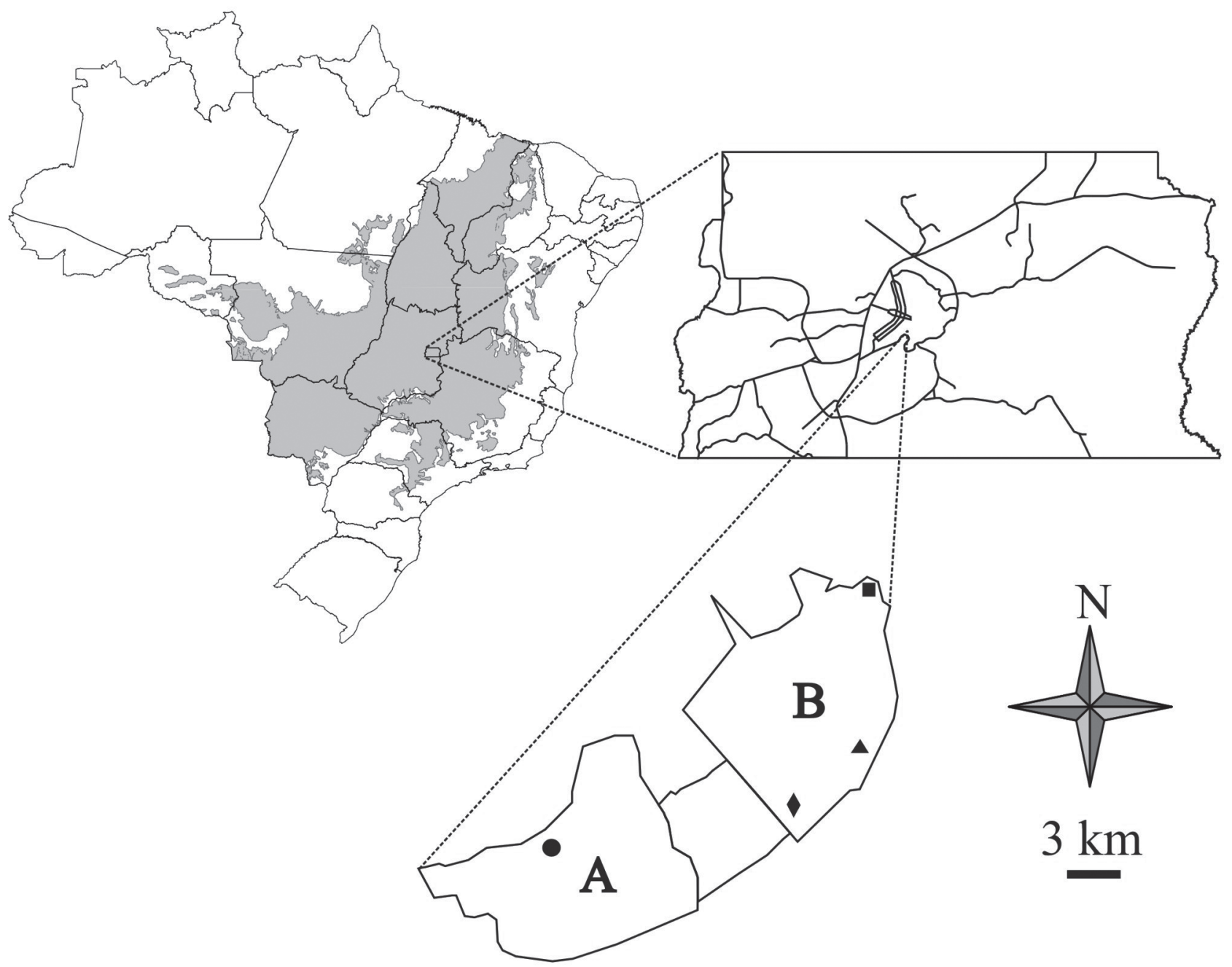

Figura 1. Mapa contendo as localizações das duas áreas de estudo em Brasília, DF, no Brasil central, onde (A) representa a Fazenda Água Limpa (FAL) e (B) o Jardim Botânico de Brasília (JBB). A área em cinza no mapa do Brasil representa a distribuição do bioma Cerrado. O circulo representa localização do fragmento de cerradão amostrado da FAL, o quadrado, o triângulo e o losango representam os três fragmentos de cerradão localizados no JBB (JB1, JB2 e JB3).

características da genitália, não avaliamos esta condição. Verificamos a idade de acordo com os padrões de erupção dentária segundo Pinheiro et al. (2002).

\section{Coleta de fezes}

Coletamos as fezes dos indivíduos capturados diretamente das armadilhas e/ou no momento de manipulação de cada indivíduo. Cada vez que um animal era capturado na armadilha, esta era limpa no local para não haver qualquer tipo de contaminação ao se coletar as fezes de outros indivíduos que caíssem posteriormente na mesma armadilha. Para uma maior independência amostral, utilizamos apenas uma amostra de cada indivíduo por época do ano (seca ou chuvosa). Cada amostra fecal foi lavada em duas peneiras de diferentes malhas $(0,1 \mathrm{~mm}$ e $0,7 \mathrm{~mm})$ sobrepostas (Leiner \& Silva 2007). Foi visualizado o conteúdo encontrado nas fezes com auxílio de uma lupa estereoscópica binocular para a detecção de itens derivados de frutos tais como sementes, polpa ou fibra. As sementes encontradas nas fezes foram contabilizadas e separadas para posterior identificação e germinação. Identificamos as sementes a partir de uma coleção de referência de frutos coletados no local e/ou com $\mathrm{o}$ auxílio especialistas.

\section{Germinação}

A germinação das sementes após a passagem pelo trato digestório de G. agilis foi avaliada. Para isso, separamos sementes das amostras de fezes e colocamos em placas de Petri contendo algodão umedecido. De maneira similar, colocamos para germinar sementes retiradas diretamente dos frutos das mesmas espécies encontradas nas fezes, como grupo controle (Lessa \& Costa 2009). Quando possível, utilizamos como padrão uma proporção de 10 sementes do controle, para cada semente encontrada nas fezes.

Uma das principais fontes de quebra de dormência para plantas tropicais, principalmente para plantas pioneiras ou de estágio secundário, é a incidência de luz (Gómes-Pompa \& Vázquez-Yanes, 1981). Desta forma, submetemos as se- 
mentes de cada tratamento contidas nas placas de Petri à luz artificial proveniente de lâmpadas fluorescentes $(40 \mathrm{~W}) \mathrm{em}$ intervalos de 12:12 h. Utilizamos um timer programado para acender as lâmpadas de 6:00 h às 18:00 h. A cada dois dias contabilizávamos as sementes germinadas, por um período de dois meses consecutivos. Com isso pudemos manter os dois grupos, tanto as sementes das fezes quanto as sementes do grupo controle em condições ambientais idênticas.

\section{Disponibilidade de frutos}

Para estimar a disponibilidade dos frutos ao longo do ano, estabelecemos aleatoriamente, em cada grade de captura, oito transecções de $20 \times 5 \mathrm{~m}$. Em cada sessão de captura, contabilizamos, no interior das transecções, o número de frutos produzidos por todos os indivíduos reprodutivos encontrados. Embora didelfídeos tendam a se alimentar mais frequentemente de frutos carnosos e com sementes de pequeno porte (Medellín 1994, Cáceres \& Monteiro-Filho 2007; Lessa \& Costa 2009), optamos por não selecionar a priori nenhuma espécie ou família de planta para a contagem de frutos disponíveis no ambiente, realizando uma contagem geral de todos os frutos avistados. Adicionalmente, como forma de obter uma coleção de referência, os frutos de cada fragmento foram coletados e as sementes devidamente limpas, identificadas e acondicionadas em potes.

\section{Análises Estatísticas}

Para verificar possíveis diferenças entre a germinação das sementes retiradas das amostras fecais e das sementes retiradas diretamente dos frutos, realizamos um teste do Qui-quadrado. Os valores obtidos de germinabilidade (i.e., número de sementes germinadas e número de sementes que não germinaram) foram agrupados por espécie de planta, pois todas as amostras foram submetidas à mesma metodologia durante o período do estudo. Quando possível, avaliamos diferenças intraespecíficas (sexo e condição reprodutiva) e sazonais (estação chuvosa e seca), no consumo de frutos por G. agilis. Para tal, realizamos um Teste- $t$ por reamostragem com 10.000 repetições. Também utilizamos uma ANOVA fatorial, por reamostragem, para avaliarmos possíveis variações sazonais e entre sexos, além das respectivas interações entre esses fatores. Consideramos, para essa análise, somente as sementes do grupo "Miconia spp". Espécies desse gênero produziram frutos nas duas estações do ano e apresentam sementes semelhantes em tamanho, formato e quantidade por fruto (N.F. Camargo, obs. pess.). Essas características possibilitaram o agrupamento dessas sementes para a análise bem como comparações sazonais. Uma vez que fêmeas reprodutivas só foram capturadas na estação chuvosa, e pela dificuldade em se verificar a condição reprodutiva dos machos, não foi possível avaliar a interação entre época do ano, sexo e condição reprodutiva. Portanto, comparamos a quantidade de sementes nas fezes de fêmeas reprodutivas e não reprodutivas separadamente, por meio de um Teste- $t$ por reamostragem. Além disso, as análises estatísticas comparan- do fêmeas reprodutivas com fêmeas não reprodutivas ou com machos foram realizadas somente com amostras coletadas na época chuvosa, para desconsiderar qualquer efeito temporal na disponibilidade de recursos. Tanto para o Teste- $t$ como para a ANOVA fatorial por reamostragem, o número total de sementes por amostra fecal foi utilizado como variável dependente e os fatores intraespecíficos e sazonais utilizados como variáveis categóricas independentes.

Também verificamos se G. agilis consumia os frutos na mesma proporção em que os mesmos ocorriam no ambiente ou se a espécie estaria selecionando determinados frutos. Uma vez que representantes da família Melastomataceae eram abundantes nos ambientes estudados, além do que dados preliminares indicaram a ocorrência frequente de sementes desse grupo nas fezes de G. agilis, optamos por restringir as análises a uma possível seleção por frutos dessa família. Desta forma, comparamos a incidência de sementes de Melastomataceae nas fezes com o total de sementes pertencentes a qualquer outra família de planta, cujas espécies tivessem produzido frutos nos sítios amostrados. Para essa comparação, utilizamos o teste do Qui-quadrado. Para esse teste, consideramos como valores esperados o número de sementes desses dois grupos de plantas (melastomatáceas comparadas com outras famílias) de acordo com a proporção de cada um desses grupos contabilizada em campo. Os valores observados foram os totais de sementes de cada um desses dois grupos encontrados nas fezes. Ao realizarmos uma ANOVA fatorial utilizando como variável dependente o número de frutos, e sítios e campanhas como variáveis independentes, não verificamos diferença entre sítios $(F=1,00$; g.l. $=3 ; P=0,392)$ ou entre campanhas $(F=1,95$; g.l. $=5$; $P=0,083)$. Contudo, verificamos interação significativa entre sítios e campanhas $(F=1,84$; g.l. $=15 ; P=0,025)$. Desta forma, optamos por realizar o teste de Qui-quadrado para investigar o consumo de frutos, de acordo com a disponibilidade, para cada sitio amostrado (FAL, JB1, JB2 e JB3 - Fig. 1) e para cada estação do ano (seca e chuvosa) separadamente.

\section{Resultados}

\section{Germinação}

Encontramos sementes de oito espécies de plantas em um total de 186 amostras de fezes $(44,1 \%$ das amostras totais): 48 amostras da FAL (Chuva $=41$; Seca=7), 69 da JB1 (Chuva $=33$; Seca=36), 53 da JB2 (Chuva $=40$; Seca=13) e 16 da JB3 (Chuva=13; Seca=3). Dessas espécies, cinco eram da família Melastomataceae, uma espécie não identificada da família Solanaceae (daqui em diante, Solanaceae sp. 1) e uma da família Viscaceae. Sementes do gênero Miconia foram a mais frequentemente encontradas nas fezes (Tab. 1).

Os testes de germinação comparativos foram realizados para o grupo "Miconia spp." (incluindo três espécies do gênero, Tab. 1), M. cuspidata, O. congestiflora e P. perrottetii (Tab. 2). Uma vez que nem sempre foi possível identificar 
Tabela 1. Frequência, hábito e família das espécies vegetais cujas sementes foram encontradas em 186 amostras fecais de Gracilinanus agilis obtidas entre setembro de 2009 e agosto de 2010 em fragmentos de Cerradão em região de Cerrado no Brasil central. Também está indicada a época do ano em que encontramos frutos disponíveis dessas espécies nos sítios amostrados.

\begin{tabular}{|c|c|c|c|c|}
\hline Espécie & Frequência (\%) & Forma de vida & Frutificação & Família \\
\hline Miconia spp. ${ }^{*}$ & 44,28 & Arbusto & Chuvosa & Melastomataceae \\
\hline Miconia cuspidata & 23,12 & Árvore & Seca & Melastomataceae \\
\hline Miconia pepericarpa & 1,08 & Árvore & Seca & Melastomataceae \\
\hline Ossaea congestiflora & 3,03 & Arbusto & Chuvosa & Melastomataceae \\
\hline Solanaceae sp. 1 & 12,90 & $* *$ & Chuvosa & Solanaceae \\
\hline Phoradendron perrottetii & 15,59 & Parasita & Chuvosa & Viscaceae \\
\hline
\end{tabular}

*inclui M. albicans, M. ferruginata, M. pohliana.

** espécie não identificada

Tabela 2. Resultados obtidos na comparação da germinação das sementes encontradas em amostras fecais de Gracilinanus agilis e daquelas obtidas de frutos coletados nos fragmentos de cerradão amostrados no Cerrado do Brasil central.

\begin{tabular}{|c|c|c|c|c|c|c|c|}
\hline \multirow[b]{2}{*}{ Espécie } & \multicolumn{2}{|c|}{ Fezes } & \multicolumn{2}{|c|}{ Controle } & \multirow[b]{2}{*}{$X^{2}$} & \multirow[b]{2}{*}{$P$} & \multirow[b]{2}{*}{ g.l. } \\
\hline & $\begin{array}{c}\text { Sementes } \\
\text { testadas }\end{array}$ & $\begin{array}{c}\text { Germinação } \\
(\%)\end{array}$ & $\begin{array}{l}\text { Sementes } \\
\text { testadas }\end{array}$ & $\begin{array}{c}\text { Germinação } \\
(\%)\end{array}$ & & & \\
\hline Miconia spp. ${ }^{*}$ & 87 & 23,00 & 870 & 24,02 & 0,046 & 0,829 & 1 \\
\hline Miconia cuspidata & 222 & 16,21 & 2220 & 20,63 & 2,437 & 0,118 & 1 \\
\hline Ossaea congestiflora & 29 & 10,34 & 290 & 63,10 & 30,186 & $<0,0001^{*}$ & 1 \\
\hline Phoradendron perrottetii & 110 & 50,90 & 850 & 51,88 & 0,037 & 0,847 & 1 \\
\hline Todas as sementes & 448 & 25,67 & 4230 & 30,52 & 0,558 & 0,455 & 1 \\
\hline
\end{tabular}

${ }^{*}$ Resultado significativo $(\mathrm{P}<0,05)$

em nível de espécie as sementes de Miconia spp., já que algumas vezes a turgidez e coloração variavam após a passagem pelo trato digestório do animal, optamos por realizar o teste utilizando como controle uma mistura das sementes destas espécies colocadas para germinar. O teste de germinação não foi realizado para $M$. pepericarpa, uma vez que não foi obtido um número suficiente de sementes nas fezes dos animais $(\mathrm{N}=2)$. Apesar de termos encontrado uma quantidade representativa de sementes de Solanaceae sp. 1 nas fezes $(\mathrm{N}=71)$, os frutos dessas sementes não foram encontrados nos sítios amostrados.

Contrariamente às nossas expectativas, não encontramos diferenças estatísticas na germinação entre as sementes do controle e as sementes encontradas nas fezes de G. agilis para Miconia spp., P. perrottetii e M. cuspidata. Entretanto, obtivemos um maior percentual de germinação das sementes do controle de O. congestiflora (Tab. 2).

\section{Variação intraespecífica no consumo de frutos}

Os testes realizados com objetivo de verificar potenciais variações intraespecíficas para G. agilis em relação ao consumo e potencial dispersão de sementes foram realizados para quatro espécies ou grupo de plantas: $P$. perrottetii, Solanaceae sp. 1, M. cuspidata e Miconia spp. O baixo número de indivíduos de $G$. agilis que consumiram $M$. pepericarpa $(\mathrm{N}=2)$ e $O$. congestiflora $(\mathrm{N}=6)$ não permitiu uma análise intraespecífica para essas espécies.
Não encontramos diferenças estatísticas entre machos e fêmeas quanto ao consumo de sementes de $P$. perrottetii, Solanaceae sp. 1 e M. cuspidata. Contudo, fêmeas consumiram maior quantidade de sementes de Miconia spp. do que machos. Além disso, não detectamos diferenças no consumo de sementes entre fêmeas reprodutivas e não reprodutivas para Solanaceae sp. 1. Para Miconia spp, no entanto, o consumo de sementes por fêmeas reprodutivas foi significativamente maior (Tab. 3). A comparação entre sexo e condição reprodutiva não foi realizada para todas as espécies de plantas, uma vez que não obtivemos número de indivíduos suficientes para comparações estatísticas. Além disso, a comparação no consumo de sementes por $G$. agilis por espécie de planta consumida não foi realizada por estação do ano, pois as espécies não frutificaram em ambas as estações consideradas (Tab. 1).

A avaliação da variação intraspecífica no número total de sementes de Miconia tanto da estação seca quanto da chuvosa encontradas nas fezes indicou que fêmeas de G. agilis defecam maior quantidade de sementes (média $\pm \mathrm{EP}=21,7 \pm 3,8$ ) em comparação com os machos $(14,4 \pm 3,0)(F=26,32 ; P<0,0001)$, o que está de acordo com nossas expectativas. $O$ fator estação do ano também foi significativo $(F=452,22 ; P<0,0001)$ e existiu interação entre sexo e sazonalidade $(F=30,10 ; P<0,0001)$. Tanto fêmeas como machos parecem aumentar o consumo de frutos com sementes na época seca (contrariando nossa segunda predição), contudo, fêmeas apresentam mais sementes nas fezes 
Tabela 3. Resultados obtidos para as comparações (teste-t por reamostragem) entre os valores médios da quantidade de sementes obtidas nas amostras de fezes de grupos de indivíduos de Gracilinanus agilis. Fizemos comparações independentes para cada espécie de planta consumida pelo marsupial. Nas análises, não consideramos as épocas seca e chuvosa uma vez que as diferentes espécies de plantas apresentaram frutos somente em uma das estações do ano (ver Tabela 1). Med = número médio de sementes encontradas nas amostras de fezes. $\mathrm{EP}=$ Erro padrão. $\mathrm{N}=$ Numero de amostras para cada grupo.

\begin{tabular}{|c|c|c|c|c|}
\hline Comparação & Med & EP & $\mathrm{N}$ & $P$ \\
\hline \multicolumn{5}{|c|}{ Miconia spp. } \\
\hline Machos & 5,8 & 1,5 & 21 & \multirow{2}{*}{$0,048^{*}$} \\
\hline Fêmeas & 17,3 & 3,3 & 66 & \\
\hline Fêmeas reprodutivas & 24,7 & 6,6 & 28 & \multirow{2}{*}{$0,031^{\star}$} \\
\hline Fêmeas não reprodutivas & 13,0 & 2,8 & 38 & \\
\hline Machos & 5,8 & 1,5 & 21 & \multirow{2}{*}{0,077} \\
\hline Fêmeas não reprodutivas & 13,0 & 2,8 & 38 & \\
\hline Machos & 5,8 & 1,5 & 21 & \multirow{2}{*}{$0,021^{\star}$} \\
\hline Fêmeas reprodutivas & 24,7 & 6,6 & 28 & \\
\hline \multicolumn{5}{|c|}{ Miconia cuspidata } \\
\hline Machos & 35,1 & 10,6 & 21 & \multirow{2}{*}{0,070} \\
\hline Fêmeas & 24,3 & 10,8 & 22 & \\
\hline \multicolumn{5}{|c|}{ Solanaceae sp. 1} \\
\hline Machos & 1,7 & 1,1 & 7 & \multirow{2}{*}{0,175} \\
\hline Fêmeas & 3,3 & 3,2 & 19 & \\
\hline Fêmeas reprodutivas & 4,4 & 4,0 & 12 & \multirow{2}{*}{0,147} \\
\hline Fêmeas não reprodutivas & 2,5 & 2,5 & 7 & \\
\hline \multicolumn{5}{|c|}{ Phoradendron perrottetii } \\
\hline Machos & 12,6 & 4,1 & 9 & \multirow{2}{*}{0,199} \\
\hline Fêmeas & 30,0 & 9,4 & 10 & \\
\hline
\end{tabular}

${ }^{*}$ Resultado significativo $(\mathrm{P}<0,05)$

do que machos na estação chuvosa (Fig. 2). O teste a posteriori de Tukey indicou que o consumo entre machos e fêmeas não diferiu significativamente na estação da seca, porém diferiu na época chuvosa. Não verificamos diferenças entre fêmeas reprodutivas $(20,4 \pm 5,6)$ e não reprodutivas $(16,7 \pm 3,5)(P=0,112)$.

\section{Seleção de frutos}

Nossos resultados indicaram que, no geral, G. agilis, selecionou positivamente espécies da família Melastomataceae nas duas épocas do ano. Na época chuvosa, a proporção observada de sementes dessa família foi maior do que a esperada em dois fragmentos (JB1: $\chi^{2}=31,48$, g.l. $=1$, $P=<0,001$ e JB2: $\chi^{2}=65,10$, g.l. $\left.=1, P<0,001\right)$ e para os outros dois não houve diferenças significativas. Esse padrão foi ainda mais evidente na época seca, quando encontramos somente sementes de Melastomataceae nas amostras de fezes, resultando em seleção significativa em três dos quatro sítios (FAL: $\chi^{2}=27,86$, g.l. $=1, P<0,0001$; JB1: $\chi^{2}=5,80$, g.l. $\left.=1, P=0,001 ; \mathrm{JB} 2: \chi^{2}=6,51, g . l .=1, P=0,001\right)$ e ausência de diferença significativa no fragmento remanescente (Fig. 3).

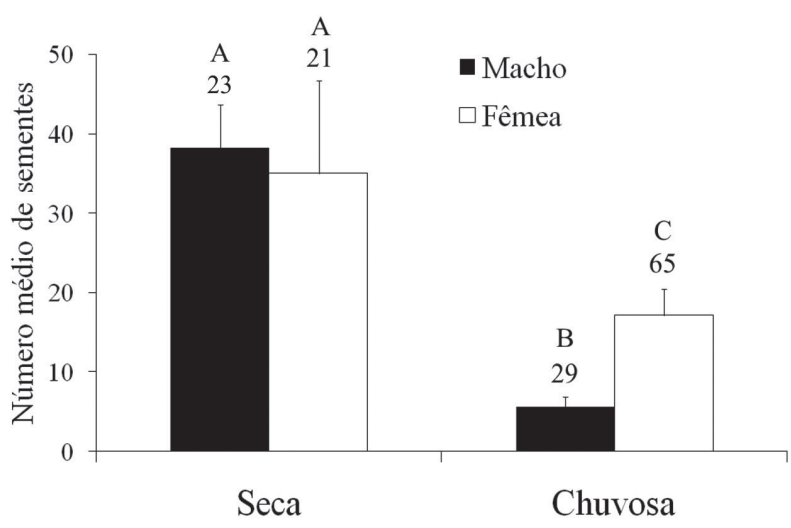

Figura 2. Comparação entre machos e fêmeas nas épocas seca e chuvosa no número médio de sementes de Miconia encontradas nas amostras de fezes de Gracilinanus agilis. As barras acima das colunas representam o erro padrão. Os números acima das barras indicam o número de indivíduos analisados. Letras distintas representam diferenças significativas $(P<0,05)$ de acordo com a ANOVA fatorial por reamostragem.

\section{Discussão}

Nossos dados mostraram que, de forma geral, as sementes dos frutos consumidos por G. agilis não apresentam maior taxa de germinação após a passagem pelo trato digestório desse animal, em comparação com as sementes dos controles. Contudo, a espécie $O$. congestiflora apresentou menor germinabilidade após o consumo dos frutos. É possível que as sementes dessa espécie por serem as menores encontradas neste estudo (0,35-0,5 mm; Silva \& Romero 2008), apresentem maior chance de serem danificadas pelo ácido clorídrico e enzimas que compõe o suco gástrico de G. agilis (van der Pijl 1972; Murray et al. 1994). Parece improvável que as sementes tenham sido danificadas pelos dentes do animal ao consumir os frutos, uma vez que não encontramos nenhuma semente fragmentada desta espécie nas amostras fecais. As sementes de Miconia spp. encontradas neste estudo possuem tamanhos que variam de 0,5 a 1,5 mm. Lessa \& Costa (2009) sugerem que sementes com tamanho aproximado de $1 \mathrm{~mm}$ tendem a permanecer intactas quando consumidas por marsupiais de pequeno porte, como é o caso de G. agilis. Contudo, nossos dados sugerem que sementes muito pequenas também podem ser danificadas.

O aumento na germinação de sementes após o consumo de frutos por possíveis dispersores não é uma regra geral (Cantor et al. 2010). Uma série de motivos pode ser responsável pela inexistência de qualquer efeito na germinabilidade ou mesmo por uma menor taxa de germinação. A intensidade e qualidade luminosa (Cáceres \& Monteiro-Filho 2007), o tipo de fruto, o tamanho das sementes, forma de vida da planta, zona climática em que as plantas ocorrem, condições experimentais ou o táxon do frugívoro estão entre as principais variáveis que influenciam na quebra de dormência das sementes e germinabilidade (Traveset 1998; Traveset \& Verdú 2002). No nosso estudo, não encontramos indícios de que a passagem pelo trato digestório beneficia a germinabilidade de nenhuma das espécies testadas. Esses resultados divergem do estudo de Lessa \& Costa (2009), que indicou que sementes de duas melastomatáceas (Clidemia 
Melastomataceae

n.s.

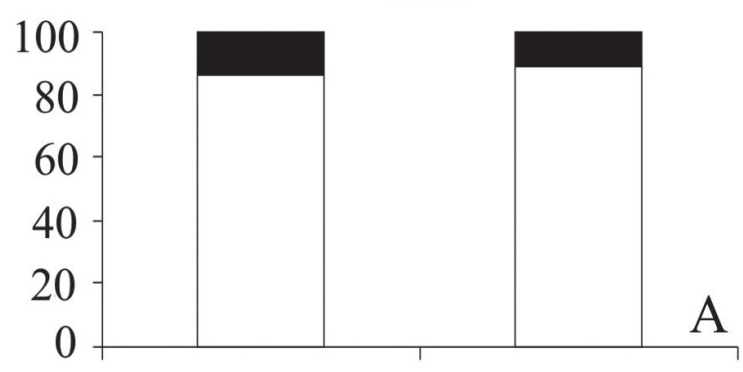

JB1

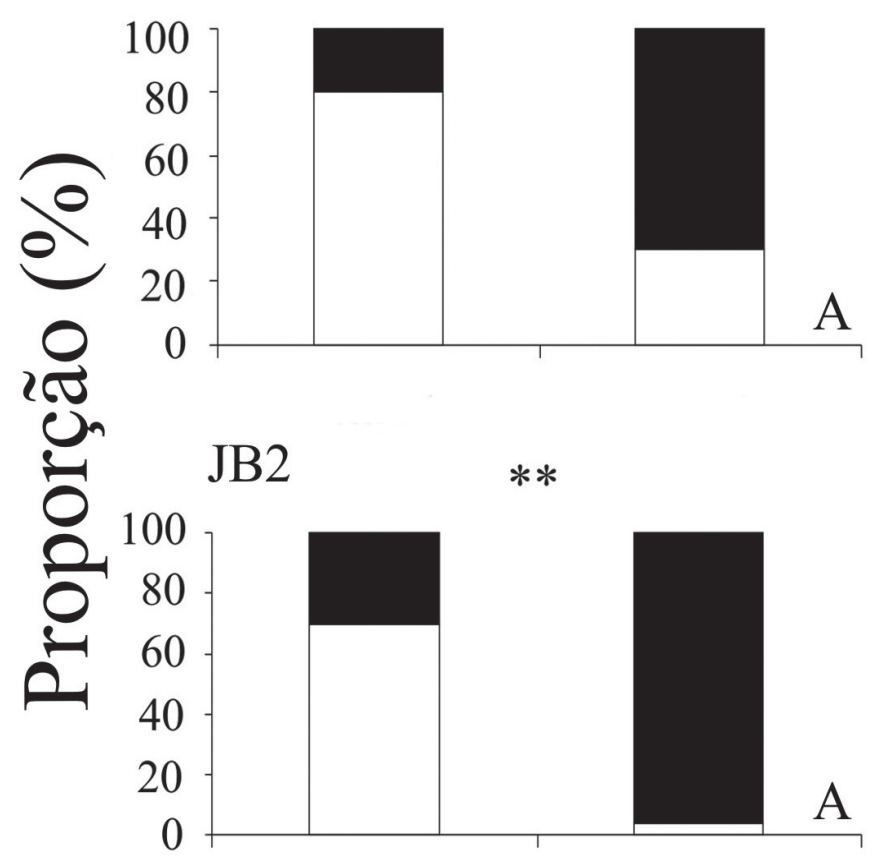

JB3

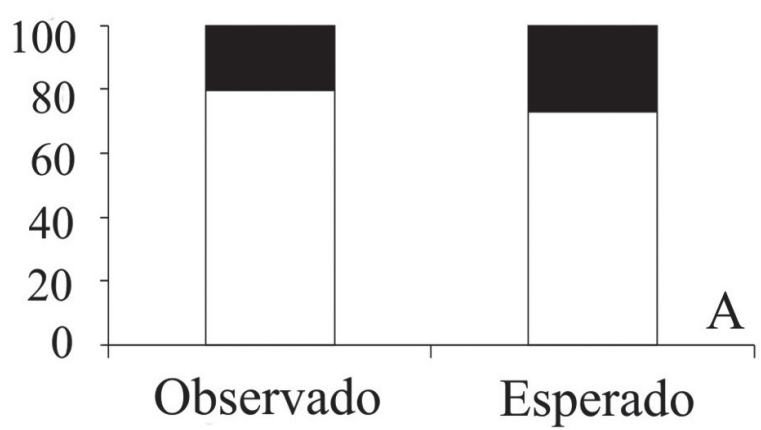

D Outras Famílias

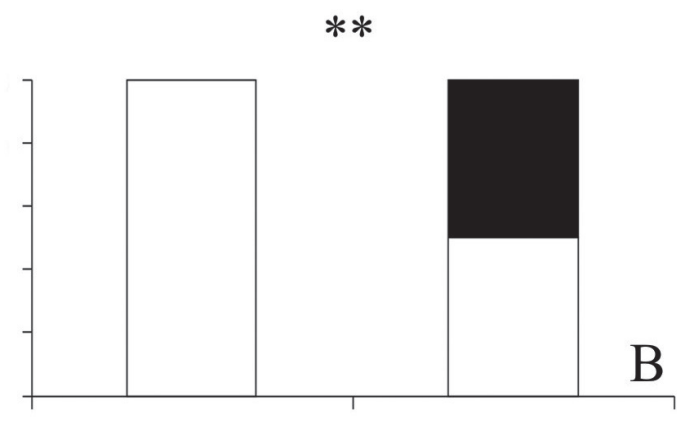

*

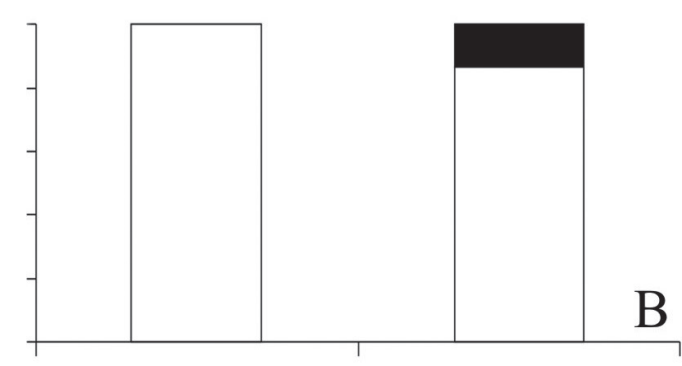

*

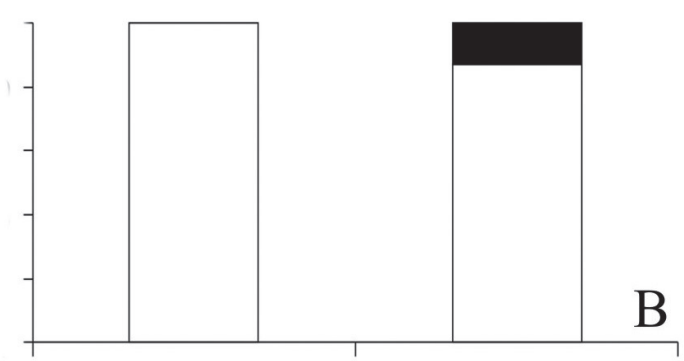

n.s.

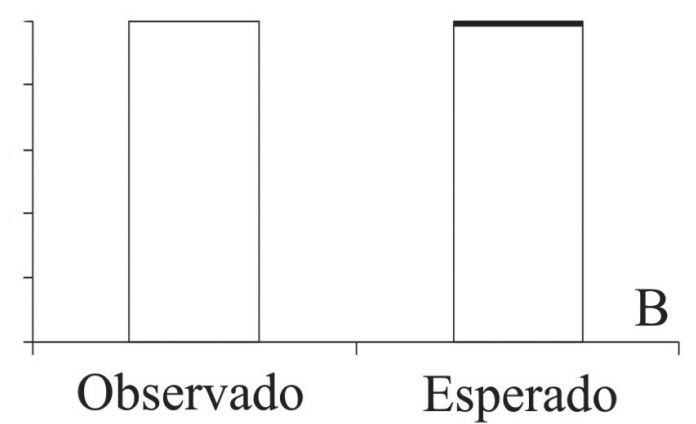

Figura 3. Proporções de frutos em cada sítio amostrado de acordo com o que foi encontrado nas fezes de G. agilis (observado) e a disponibilidade de acordo com a contagem de frutos em campo (esperado) nas épocas chuvosa e seca. Diferenças significativas entre o observado e o esperado são indicadas por “»” $(P<0,05)$ ou “**” $(P<0,001)$ de acordo com o teste de Qui-quadrado. O código "n.s." indica resultados não significativos. Código dos sítios como na Fig. 1.. 
urceolata DC. e uma espécie não identificada) encontradas nas fezes de G. agilis em Cerrado de São Paulo apresentaram maior germinabilidade após a passagem pelo trato digestório do animal. Essa divergência pode ser devido às diferenças entre espécies consumidas ou então ter sido um artefato devido ao pequeno tamanho amostral dos experimentos de Lessa \& Costa (número de sementes amostradas variou de 10 a 45 por tratamento).

Em comparação com outros estudos para o gênero Gracilinanus em Cerrado, nossos dados revelaram uma importância relativamente alta no consumo de frutos por G. agilis. Cerca de $45 \%$ das amostras totais de fezes continham sementes. Se levarmos em consideração outros itens tais como fibra e polpa derivados de frutos, a freqüência aumenta para aproximadamente $86 \%$. Da mesma forma, se consideramos a polpa e fibras derivadas dos frutos de $\mathrm{Me}$ lastomataceae, cuja coloração é tipicamente azul-arroxeada, a freqüência de consumo para esta família aumenta para cerca de $82 \%$. Esses são os maiores valores encontrados para freqüência no consumo de frutos não só para a espécie G. agilis, como também para esse gênero de marsupial (ver Martins et al. 2006; Lessa \& Costa 2009; Bocchiglieri et al. 2010). Nossos dados parecem estar mais de acordo com os estudos realizados por Astúa de Moraes et al. (2003) e Santori \& Astúa de Moraes (2006), que verificaram um alto consumo de frutos por G. agilis em laboratório.

Em relação aos frutos consumidos por G. agilis, verificamos que esse marsupial consome basicamente frutos de plantas pioneiras, as quais estiveram presentes em $84,4 \%$ das fezes que continham sementes. A família Melastomataceae, principalmente o gênero Miconia, parece ser um importante componente na dieta do animal, tendo ocorrido em cerca de $68,5 \%$ das amostras de fezes que continham sementes. Essa alta ocorrência de melastomatáceas não foi registrada em outros estudos no Cerrado sobre dieta de Gracilinanus spp., com as maiores freqüências de ocorrência para espécies da família variando entre 2,3\% (Miconia sp., Martins et al. 2006) e 5,3\% (Clidemia urceolata, Lessa \& Costa 2009). Outros marsupiais de Cerrado apresentaram uma freqüência entre 5 e $20 \%$ no consumo de Miconia holosericea (L.) DC (Lessa \& Costa 2009).

Os dados que obtivemos em relação à freqüência de consumo e quantidade de sementes viáveis nas fezes de espécies tidas como pioneiras reforça a idéia de que G. agilis pode desempenhar um papel relevante na recuperação de áreas degradadas (Pinheiro et al. 2002; Cáceres 2002; Leiner \& Silva 2007; Lessa \& Costa 2009). Isso considerando o fato de que indivíduos dessa espécie aparentemente não danificam as sementes consumidas de plantas pioneiras, com exceção de O. congestiflora. Mesmo que as espécies de planta encontradas neste estudo não apresentem maior germinabilidade após o consumo dos frutos, é razoável assumir que as sementes tenham maior probabilidade de germinação e estabelecimento quando depositadas a maiores distâncias da planta matriz pelo marsupial estudado (Janzen 1970; Connell 1971; Howe et al. 1985).

O presente estudo aparentemente representa o primeiro registro de um marsupial se alimentado da erva-de-passarinho $P$. perrottetii, uma espécie de planta hemiparasita. A grande maioria das espécies de erva-de-passarinho depende das aves para a dispersão de suas sementes, sendo algumas destas dispersoras altamente especializadas no consumo dos frutos (Reid 1991). Até o momento, somente um outro registro desta natureza foi realizado na América do sul por Amico \& Aizen (2000). Os autores verificaram uma estreita relação de mutualismo entre o marsupial Dromiciops australis (Thomas, 1894), endêmico do sul da América do Sul, e um outro tipo de erva-de-passarinho Tristerix corymbosus (L.) Kuijt. - ao apontar o marsupial como o exclusivo dispersor das sementes dessa planta. Adicionalmente, esses autores verificaram maior germinação das sementes após a passagem pelo trato digestório de $D$. australis.

A dispersão de sementes de erva-de-passarinho é um evento particularmente crítico para a espécie, uma vez que as sementes precisam ser depositadas em galhos de plantas hospedeiras suscetíveis, para que haja alguma chance de estabelecimento (Monteiro et al. 1992, Sargent 1995). No presente estudo, aproximadamente $15 \%$ das amostras de fezes de $G$. agilis continham sementes de $P$. perrottetii. Por mais que os principais dispersores deste gênero sejam possivelmente pássaros (Cazetta \& Galetti 2007), é possível que G. agilis forneça algum efeito positivo no estabelecimento desta espécie, uma vez que este marsupial utiliza consideravelmente o estrato vertical do sub-bosque (Nitikman \& Mares 1987) e pode depositar as sementes em galhos de possíveis hospedeiros.

Frutos possuem alto valor calórico e podem ter grande relevância na época em que as fêmeas estão gestantes ou lactantes. Os frutos de algumas espécies de Miconia são constituídos de 70 a $80 \%$ de água e cerca de 12 a $18 \%$ de açúcar, porém com quantidades relativamente baixas de proteína (entre 1 e 2\%) (Maruyama et al. 2007). Encontramos indicativos claros de diferenças intra-específicas no consumo de frutos de Miconia spp. por G. agilis. O fato das fêmeas apresentarem maior consumo destes frutos em relação aos machos parece ser devido ao maior consumo destes frutos por fêmeas reprodutivas. Isso porque o número de sementes não variou significativamente entre amostras de machos e fêmeas não-reprodutivas, contudo, houve diferença entre machos e fêmeas reprodutivas. O maior consumo de frutos por ambos os sexos na estação seca, detectado no presente estudo, pode ser devido a uma menor abundância relativa de invertebrados nessa época (Pinheiro et al. 2002), o que faria com que os marsupiais dependessem mais de frutos nessa época de ano.

Além da menor disponibilidade de outros recursos, frutos podem ser importantes para G. agilis na época seca também como fonte de água. O cerradão é um tipo de fisionomia que não está associada a cursos de água (Ribeiro \& Walter 1998) e esse recurso pode ser um fator extremamente limitante para os animais nos meses de seca. Estudos já publicados que analisaram a água metabólica produzida e perda evaporativa da água indicam que $G$. agilis não possui nenhum tipo de adaptação fisiológica para economia de água (Cooper et al. 2009). O aumento no consumo de frutos na estação seca pode ser, portanto, devido ao potencial dos frutos em fornecer água 
para os animais. $\mathrm{O}$ consumo de frutos por outros marsupiais neotropicais como Didelphis albiventris (Lund, 1840) (Santori et al. 2004) e Caluromys lanatus (Olfers, 1818) (Cáceres 2005) também foi sugerido como forma de obter água em épocas de déficit hídrico. Além disso, G. agilis possui um ceco relativamente bem desenvolvido (9,3\% do trato gastrointestinal total, N.F. Camargo, obs. pessoal), o que pode sugerir também uma adaptação morfológica para reabsorção de água em épocas de estresse hídrico, como indicado para outras sete espécies de didelfídeos neotropicais (Cáceres 2005).

Os resultados obtidos indicam também que, na época chuvosa, fêmeas se alimentam mais de frutos do que os machos. Uma maior necessidade nutricional das fêmeas nesse período, que coincide com a época reprodutiva da espécie, pode ser a razão dessa diferença (Cáceres 2002). Isto fica mais evidente quando ao comparar fêmeas reprodutivas com não reprodutivas, as reprodutivas preferiram frutos de Miconia spp. em comparação com fêmeas não-reprodutivas.

Devido ao padrão de frutificação sazonal em regiões tropicais e subtropicais (Charles-Dominique et al. 1981), didelfídeos têm sido considerados como oportunistas em relação ao consumo de frutos (Atramentowicz 1988; Medellín 1994). Contudo, nossos dados demonstraram que $G$. agilis tende a selecionar frutos de Melastomataceae. Mesmo em alguns sítios que continham baixa abundância de frutos desta família de planta, a proporção de indivíduos se alimentando de frutos foi estatisticamente maior do que o esperado. Isto sugere que este marsupial não se alimenta somente destes frutos simplesmente por serem abundantes nas áreas de forma geral.

\section{Conclusões}

Verificamos que G. agilis pode atuar como potencial dispersor de sementes, uma vez que para as quatro espécies testadas houve uma proporção de sementes que permaneceram viáveis após a passagem no trato digestório do animal e que para três dessas espécies não houve diferenças significativas na porcentagem de germinação entre sementes ingeridas e sementes não ingeridas. Esse marsupial apresenta variação sazonal e intraespecífica no consumo de frutos. Fêmeas em comparação aos machos, fêmeas reprodutivas em comparação às fêmeas não reprodutivas e indivíduos da época seca em comparação aos da época chuvosa defecam maior quantidade de sementes, sugerindo um maior consumo de frutos por esses grupos. Além disso, verificamos que G. agilis tende a selecionar a família Melastomataceae, independente da disponibilidade de frutos no seu ambiente. Especificamente, nossos dados indicaram a relevância de espécies de Miconia na dieta de G. agilis nas áreas de cerradão estudadas, especialmente na época de seca. Nessa época, o consumo de frutos além de fornecer energia também pode contribuir para satisfazer os requerimentos hídricos da espécie. Desta forma, a Melastomataceae arbórea Miconia cuspidata, cuja semente foi a mais abundante nas fezes de G. agilis na estação seca, pode ser de extrema importância para a sobrevivência da espécie em áreas de cerradão no Brasil central.

\section{Agradecimentos}

Ao Conselho Nacional de Desenvolvimento Científico e Tecnológico (CNPq) pelo financiamento para esse estudo (Edital Universal 2009, Processo no $483117 / 2009-9$ ) e também pela concessão de bolsas de Mestrado (NFC e JFR), Iniciação Científica (PIBIC - RMSC) e de Produtividade em Pesquisa (EMV, Processo no 308153/2007-3) durante a realização desse estudo. À Dra. Cássia Munhoz e aos responsáveis pelo Herbário da Universidade de Brasília pela identificação das plantas coletadas em campo.

\section{Referências Bibliográficas}

Amico, G. \& Aizen, M.A. 2000. Mistletoe seed dispersal by a marsupial. Nature 408: 929-930.

Astúa de Moraes, D.; Santori, R.T.; Grelle C.E.V. \& Cerqueira R. 2003. Nutritional and fibre content of laboratory-established diets of Neotropical opossums (Didelphidae). Pp. 229-237. In: Jones M.; Dickman C. \& Archer M. (Eds.). Predators with pouches: The biology of carnivorous marsupials. Collingwood, CSIRO Publishing.

Atramentowics, M. 1988. La frugivorie opportuniste de trois marsupiaux didelphidés de Guyane. Revue d'Ecologie (Terre et Vie) 43: 47-57.

Ballardie, R.T. \& Whelan, R.J. 1986. Masting, seed dispersal and seed predation in the cycad Macrozamia communis. Oecologia 70: 100-105.

Batalha, M.A. \& Martins, F.R. 2004. Reproductive phenology of the cerrado plant community in Emas National Park (central Brazil). Australian Journal of Botany 52: 149-161.

Bocchiglieri, A.; Mendonça, A.F. \& Campos, J.B. 2010. Diet composition of Gracilinanus agilis (Didelphimorphia, Didelphidae) in dry woodland areas of Cerrado in central Brazil. Mammalia 74: 225-227.

Cáceres, N.C. 2002. Food habits and seed dispersal by the white-eared opossum Didelphis albiventris in Southern Brazil. Studies on Neotropical Fauna and Environment 37: 1-8.

Cáceres, N.C .2005. Comparative lengths of digestive tracts of seven didelphid marsupials (Mammalia) in relation to diet. Revista Brasileira de Zoologia 22: 181-185.

Cáceres, N.C. \& Monteiro-Filho, E.L.A. 2000. The common opossum, Didelphis aurita, as a seed disperser of several plants in southern Brazil. Ciência e Cultura 52: 41-44.

Cáceres, N.C. \& Monteiro-Filho, E.L.A. 2007. Germination in seed species ingested by opossums: implications for seed dispersal and forest conservation. Brazilian Archives of Biology and Technology 50: 921-928.

Câmara, P.A.S. 2008. Musgos acrocárpicos das Matas de Galeria da Reserva Ecológica do IBGE, RECOR, Distrito Federal, Brasil. Acta Botanica Brasilica 22: 1027-1035.

Cantor, M.; Ferreira, L.A.; Silva, W.R. \& Setz, E.Z.F. 2010. Potential seed dispersal by Didelphis albiventris (Marsupialia, Didelphidae) in highly disturbed environment. Biota Neotropica 10: 45-51.

Caron, L.; Garant, Y. \& Bergeron, J.M. 1985. The effect of digestibility values of resources on the reliability of food-habit studies from fecal analyses. Canadian Journal of Zoology 63: 2183-2186.

Casella, J. \& Cáceres, N.C. 2006. Diet of four small mammal species from Atlantic forest patches in south Brazilian. Neotropical Biology Conservation 1: 5-11.

Cazetta, E. \& Galetti, M. 2007. Frugivoria e especificidade por hospedeiros na erva-de-passarinho Phoradendron rubrum (L.) Griseb. (Viscaceae). Revista Brasileira de Botânica 30: 345-351.

Charles-Dominique, P.; Atramentowics, M.; Charles-Dominique, M.; Gérard, H.; Hladik, A.; Hladik, C.M. \& Prèvost, M.F. 1981. Les mammiferes frugivores arboricoles nocturnes dê une forest guyanaise: interrelations plantes-animaux. Revue d'Ecologie (Terre et Vie) 35: 341-435.

Clergeau, P. 1992. The effect of birds on seed germination of fleshy-fruited plants in temperate farmland. Acta Oecologica 13: 679-686.

Connell, J.H. 1971. On the role of natural enemies in prevent competitive exclusion in some marine animals and rainforest trees. Pp. 289-312. 
In: Den Boer, P.J. \& Gradwell, G.R. (Eds.). Dynamic of populations. Wageningen, Pudoc

Cooper, C. E.; Withers, P.C. \& Cruz-Neto, A.P. 2009. Metabolic, ventilatory and hygric physiology of the gracile mouse opossum (Gracilinanus agilis). Physiological Biochemical Zoology 82: 152-162.

Cowan, P.E. 1990. Fruits, seeds, and flowers in the diet of brushtailed possums, Trichosurus vulpecula, in lowland podocarp/mixed hardwood forest, Orongorongo Valley, New Zealand. New Zealand Journal of Zoology 17: 549-66.

Dennis, A.J. 2003. Scatter-hoarding by musky rat-kangaroos, Hypsiprymnodon moschatus, a tropical rain forest marsupial from Australia: implications for seed dispersal. Journal of Tropical Ecology 19: 619-627.

Dubost, G. 1988. Ecology and social life of the red acouchy, Myoprocta exilis, comparison with the orange-rumped agouti, Dasyprocta leporina. Journal of Zoology 214: 107-123.

Dungan, R.J.; O'Cain, M.J.; Lopez, M.L. \& Norton, D.A. 2002. Contribution by possums to seed rain and subsequent seed germination in successional vegetation, Canterbury, New Zealand. New Zealand Journal of Ecology 26: 121-128.

Eiten, G. 1972. The Cerrado vegetation of Brazil. Botanical Review 38 201-341.

Emmons, L.H. \& Feer, F. 1990. Neotropical rainforest mammals: a field guide. Chicago, The University of Chicago Press.

Fonseca, M.S. \& Silva Júnior, M.C. 2004. Fitossociologia e similaridade florística entre trechos de Cerrado sentido restrito em interflúvio e em vale no Jardim Botânico de Brasília, DF. Acta Botanica Brasilica 18: 19-29.

Forget, P.M. 1993. Post-dispersal predation and scatter-hoarding of Dipteryx panamensis (Papilionaceae) seeds by rodents in Panama. Oecologia 94: 255-261.

Gómes-Pompa, A. \& Vázquez-Yanes, C. 1981. Successional studies of a rain forest in Mexico. Pp. 246-266. In: West, D.C.; Shugart, H. H. \& Botkin, D.B. (Eds.). Forest succession: concepts and application. New York, Springer-Verlag.

Grelle, C.E. \& Garcia, Q.S., 1999. Potential dispersal of Cecropia hololeuca by the common opossum (Didelphis aurita) in Atlantic Forest, southeastern Brazil. Revue d'Ecologie (Terre et Vie) 54: 327-332.

Howe, H.F; Schupp, E.W. \& Westley, L.C. 1985. Early consequences of seed dispersal for a Neotropical tree (Virola surinamensis). Ecology 66: 781-791.

Janzen, D.H. 1970. Herbivores and the number of tree species in tropical forests. American Naturalist 104: 501-529.

Jordano, P. 2000. Fruits and frugivory. Pp. 125-166. In: Fenner, M. (Ed.). Seeds: the ecology of regeneration in plant communities. Wallingford, CABI Publ.

Lawton, J.H. 1994. What do species do in ecosystems? Oikos 71: 367-374.

Leiner, N.O. \& Silva, W.R. 2007. Seasonal variation in the diet of the Brazilian slender opossum (Marmosops paulensis) in a montane Atlantic Forest area, southeastern Brazil. Journal of Mammalogy 88: 158-164.

Lessa, L.G. \& Costa, F.N. 2009. Diet and seed dispersal by five marsupials (Didelphimorphia: Didelphidae) in a Brazilian cerrado reserve. Mammal Biology 75: 10-16.

Mares, M.A. \& Ernest, K.A. 1995. Population and community ecology of small mammals in a gallery forest of central Brazil. Journal of Mammalogy 76: 750-768.

Marinho-Filho, J.; Rodrigues, F.H.G. \& Juarez, K.M. 2002. The Cerrado mammals: diversity, ecology, and natural history. Pp. 266-284. In: Oliveira, P.S. \& Marquis, R.J. (Eds.). The Cerrados of Brazil: Ecology and natural history of a neotropical savanna. New York, Columbia University Press.

Martins, E.G.; Bonato, V.; Pinheiro, H. P. \& Reis, S.F. 2006. Diet of the gracile mouse opossum (Gracilinanus microtarsus) (Didelphimorphia: Didelphidae) in a Brazilian Cerrado: patterns of food consumption and intrapopulation variation. Journal of Zoology 269: 21-28.

Maruyama, P.K.; Alves-Silva, E. \& Melo, C. 2007. Oferta qualitativa e quantitativa de frutos em espécies ornitocóricas do gênero Miconia (Melastomataceae). Revista Brasileira de Biociências 5: 672-674.
Medellín, R.A. 1994. Seed dispersal of Cecropia obtusifolia by two species of opossums in the selva Lacandona, Chiapas, Mexico. Biotropica 26: $400-407$

Monteiro, R.F.; Martins, R.P. \& Yamamoto, K. 1992. Host specificity and seed dispersal of Psittacanthus robustus (Loranthaceae) in south-east Brazil. Journal of Tropical Ecology 8: 307-314.

Murray, K.G.; Russel, C. S.; Picone, C. M.; Winnet-Murray, K.; Sherwood, W. \& Kuhlmann, M. L. 1994. Fruit laxatives and seed passage rates in frugivores: consequences for plant reproductive success. Ecology 75: $989-994$

Myers, N.; Mittermeier, R.A.; Mittermeier, C.G.; Fonseca, G.A.B. \& Kent, J. 2000. Biodiversity hotspots for conservation priorities. Nature 403: 853-858.

Nitikman, L.Z. \& Mares, M.A. 1987. Ecology of small mammals in a gallery forest of central Brazil. Annals of Carnagie Museum 56: 75-95.

Pimentel, D.S. \&, Tabarelli, M. 2004. Seed dispersal of the palm Attalea oleifera in a remmant of the Brazilian Atlantic Forest. Biotropica 36: 74-84.

Pinheiro, P.S.; Carvalho, F.M.V.; Fernandez, F.A.S. \& Nessimian, J.L. 2002. Diet of the marsupial Micoureus demerarae in small fragments of Atlantic Forest in Southeastern Brazil. Studies on Neotropical Fauna and Environment 37: 213-218.

Price M.V. \& Jenkins S.H. 1986. Rodents as seed consumers and dispersers. Pp. 123-83. In: Murray D.R. (Ed.). Seed Dispersal. Sydney, Academic Press.

Reid, N. 1991. Coevolution of mistletoes and frugivorous birds. Austral Journal of Ecology 16: 457-469.

Ribeiro, J.F. \& Walter, B.M.T. 1998. Fitofisionomias do Cerrado. Pp. 87166. In: Sano, S.M. \& Almeida, S.P. (Eds.). Cerrado: ambiente e flora. Planaltina, EMBRAPA-CPAC.

Rose, K.D. 1987. Climbing adaptations in the early eocene mammal Chriacus and the origin of artiodactyla. Science 236: 314-316.

Rossi, R.V.; Bianconi, G.V. \& Pedro, W.A. 2006. Ordem Didelphimorphia. Pp. 27-66. In: Reis, N.R.; Peracchi, A.L.; Pedro, W.A. \& Lima, I.P. (Eds.). Mamíferos do Brasil. Londrina, Imprensa da UEL.

Santori, R.T. \& Astúa de Moraes, D. 2006. Alimentação, nutrição e adaptações alimentares de marsupiais brasileiros. Pp. 241-254. In: Cáceres, N.C. \& Monteiro-Filho, E.L.A. (Eds.). Os marsupiais do Brasil: biologia, ecologia e evolução. Campo Grande, UFMS Ed.

Santori, R.T.; Astúa de Moraes, D. \& Cerqueira, R. 2004. Comparative gross morphology of the digestive tract in ten Didelphidae marsupial species. Mammalia 68: 27-36.

Sargent, S. 1995. Seed fate in a tropical mistletoe: the importance of host twig size. Functional Ecology 9: 127-204.

Silva, M.A.O \& Romero, R. 2008. Melastomataceae das serras do município de Delfinópolis, Minas Gerais, Brasil. Rodriguésia 59: 609-647.

Smythe, N. 1978. The natural history of the Central American agouti (Dasyprocta punctata). Smithsonian Contributions to Zoology 257: 1-52.

Theimer, T. H. 2001. Seed scatter-hoarding by white-tailed rats: consequences for seedling recruitment by an Australian rain-forest tree. Journal of Tropical Ecology 17: 177-189.

Traveset, A. 1998. Effect of seed passage through vertebrate frugivores' guts on germination: a review. Perspectives in Plant Ecology, Evolution and Systematics 1/2: 151-190.

Traveset, A. \& Verdú, M. 2002. A meta-analysis of the effect of gut treatment on seed germination. Pp. 339-350. In: Levey, D.J.; Silva W.R. \& Galetti, M. (Eds.). Seed dispersal and frugivory: ecology, evolution and conservation. Wallingford, $\mathrm{CAB}$ International Publishing.

van der Pijl, L. 1982. Principles of dispersal in higher plants. Berlin, Springer Verlag.

Vander Wall, S.B. 2002. Masting in animal-dispersed pines facilitates seed dispersal. Ecology 83: 3508-3516.

Wiesbauer, M.B.; Giehl, E.L.H. \& Jarenkow, J.A. 2008. Morphological patterns of diaspores from animal-dispersed tree and treelet species at Parque Estadual de Itapuã, Rio Grande do Sul State, Brazil. Acta Botanica Brasilica 22: 425-435.

Willson, M. F. 1993. Mammals as seed-dispersal mutualists in North America. Oikos 67: 159-176. 